\title{
Response to Rituximab after Failure of Cyclophosphamide in the Induction Treatment in a Patient with cANCA- associated Vasculitis and Pachymeningitis: a Case Report
}

\author{
Psenak O., MD, PhD, Greil R., MD, Prof. \\ Department of Internal Medicine III with Haematology, Medical Oncology, Haemostaseology, Infectiology and \\ Rheumatology, Oncologic Centre; \\ Salzburg Cancer Research Institute - Laboratory for Immunological and Molecular Cancer Research (SCRI- \\ LIMCR), Paracelsus Medical University, Salzburg, Austria; \\ Cancer Cluster Salzburg, Salzburg, Austria \\ Address for correspondence: Rehabilitation clinic of the Paracelsus Private Medical University Salzburg \\ Salzburger Straße 520, 5084 Großgmain, Austria
}

\begin{abstract}
A 36-year-old male patient who originally presented with recurrent inflammations in the mastoid, otitis media and peripheral facial paralysis was diagnosed with sterile pachymeningitis, associated with high titres of antineutrophil cytoplasmic antibodies (cANCA) directed against proteinase 3 (PR3). Induction therapy with oral prednisolone $1 \mathrm{mg} / \mathrm{kg}$ body weight and cyclophosphamide (CYC) $750 \mathrm{mg} / \mathrm{m}^{2} \mathrm{i} . v$. every $4 \mathrm{weeks}$ was initiated. Due to side effects, increasing arthralgias and progressive meningitis after 5 doses of CYC, treatment was changed to rituximab (RTX), one cycle comprising two administrations of $1000 \mathrm{mg} R T X$ i.v. two weeks apart, repeated every 6 months. After the very first cycle of RTX, we confirmed subjective improvement of the patient's fitness as well as radiologic response. Methotrexate (MTX) was added to ease arthralgias. Painful bleeding ulcerations on finger tips were successfully treated with 22 iloprost infusions. Up to date, we have administered 7 cycles of RTX and achieved complete remission of the cANCA-associated vasculitis. After the induction therapy, maintenance treatment with $M T X$ or rituximab will be performed for at least 18-24 months.

We can demonstrate a complete remission with the use of RTX in a patient with cANCA-associated vasculitis and sterile pachymeningitis who failed to respond to the induction treatment with CYC. RTX has been well tolerated.
\end{abstract}

Keywords: cANCA-associated vasculitis; case report; pachymeningitis; cyclophosphamide; rituximab.

Contact: Oskar Psenak; psenak@europe.com

For reference: Psenak $O$, Greil R. Response to rituximab after failure of cyclophosphamide in the induction treatment in a patient with cANCAassociated vasculitis and pachymeningitis: a case report. Sovremennaya Revmatologiya=Modern Rheumatology Journal. 2021;15(4):68-71. DOI: 10.14412/1996- 7012-2021-4-68-71

\section{Introduction}

Granulomatosis with polyangiitis (GPA) is a necrotizing vasculitis affecting small and medium-sized vessels, associated with antineutrophil cytoplasmic antibodies with cytoplasmic immunofluorescence pattern (cANCA) directed against proteinase 3 (PR3). It is a very heterogeneous disease as to localization, extent, severity and clinical manifestations. It can be limited to one organ or local tissue only (nasal or oral mucosa), however it can rapidly progress to a systemic disease (ocular, lung, renal or nervous system involvement) with lethal outcome, if untreated or treated improperly. Diagnosis is made by clinical presentation, positivity of cANCA and the presence of typical histologic findings (vascular necrosis or granuloma) [1]. Local manifestations can be treated with cotrimoxazole or methotrexate [2,3], the extended disease should be treated either with cyclophosphamide (CYC) or rituximab that has been shown to be non-inferior in cANCA-positive vasculitis [4, 5]. Life-threatening conditions can be managed using plasmapheresis [6]. Induction treatment usually consists of at least 6 cycles of CYC or 3-4 cycles of rituximab $[5,7]$, accompanied by Pneumocystis jirovecii prophylaxis with cotrimoxazole due to increased prevalence of this infection in the acute phase of the disease [8]. Rituximab has also been shown to be effective in less frequent manifestations of the disease such as pachymeningitis [9]. After remission, maintenance treatment is recommended to avoid a relapse or recurrence of vasculitis.
Traditionally, two immunosuppressants used to be given: either azathioprine or methotrexate, the first is preferred in reduced glomerular filtration rate [10]. However, the MAINRITSAN trial demonstrated better outcomes using rituximab than azathioprine in the maintenance setting [11]. Maintenance treatment should last 18-24 months [12].

We present a patient with less common manifestations of ANCA-positive GPA comprising otitis media, peripheral facial palsy, orbital involvement, sterile meningitis, infiltration of the parapharyngeal tissue, pterygoid muscle as well as tissue around the internal carotid who was refractory to 5 administrations of CYC. However, after switching the induction therapy to rituximab, nearly complete remission of the above mentioned manifestations was achieved. As reports of such course of the disease are scarce, we would like to contribute to medical literature to encourage physicians to use rituximab in unusual manifestations of GPA.

\section{Case report}

A 36-year-old male patient developed tympanic cavity effusion, treated with paracentesis and drainage. This was followed by multiple operations due to recurrent otitis media; additionally a peripheral facial palsy developed on the right side. Due to recurrent fibrosing bilateral otitis, complicated by left facial palsy, mastoidectomy was performed. Atypical mycobacterial infection was assumed and antibiotic treatment with ethambu- 
КЛИНИЧЕСКИЕ НАБЛЮДЕНИЯ / CLINICAL OBSERVATIONS

tol, moxifloxacin and clarithromycin was prescribed. However, this infection was not confirmed.

Several months later, the patient presented at hospital with poor physical fitness, body weight loss of $15 \mathrm{~kg}$ and sudden decline of muscle strength in the right arm. Cerebral computed tomography (CT) showed small subdural haematoma on the right side. Additionally, right median nerve paralysis developed and the patient was transferred to the intensive care unit. Due to febrile infection with Staphylococcus aureus, detected in the blood culture, linezolid and ceftriaxone were administered. Cerebral magnetic resonance tomography (MRT) showed a thickening of the dura mater $(4 \mathrm{~mm})$ of the right hemisphere corresponding to meningitis. This condition was interpreted as a consequence of the otitis media. Transoesophageal echocardiography did not show endocarditis.

In the meantime, high titres of antineutrophil cytoplasmic antibodies with cytoplasmic immunofluorescence pattern (cANCA) directed against proteinase 3 (PR3) were detected in extended laboratory examinations (1:160). Increased inflammatory markers were detected (normal range in round brackets): leucocytes $14800 / \mu \mathrm{l}(4300-10000 / \mu \mathrm{l})$, ESR (erythrocyte sedimentation rate) $87 / 95 \mathrm{~mm} / \mathrm{h}(0-15 \mathrm{~mm} / \mathrm{h})$, CRP (C-reactive protein) $193.4 \mathrm{mg} / 1(0-5.0 \mathrm{mg} / \mathrm{l})$. Furthermore, anaemia with haemoglobin $11.8 \mathrm{~g} / \mathrm{dl}(14.0-17.5 \mathrm{~g} / \mathrm{dl})$, creatinkinase $20 \mathrm{U} / 1$ (38-174 U/l), rheumatoid factor $28 \mathrm{U} / \mathrm{ml}(0-14 \mathrm{U} / \mathrm{ml})$, creatinine $0.63 \mathrm{mg} / \mathrm{dl}(0.7-1.2 \mathrm{mg} / \mathrm{dl})$, as well as elevated liver enzymes were found.

Though histological analyses of the above mentioned inflammatory processes in the middle ear did not show granulomatous changes, the patient's condition was ascribed to cANCA-associated vasculitis with sterile meningitis as the cerebrospinal fluid did not demonstrate signs of a purulent bacterial infection. Similar cases of cANCA-associated vasculitis with otitis media have already been described [13]. Neither renal involvement (urine protein analysis) nor pulmonary manifestation (CT examination) could be found. Via gastroscopic and endosonographic examinations a $2.5 \mathrm{~cm}$ large GIST (gastrointestinal stromal tumor) in the cardia of the stomach was suspected. An extended faecal examination did not confirm parasites.

Based on these findings, induction therapy with daily oral prednisolone $1 \mathrm{mg} / \mathrm{kg}$ body weight was introduced [14]. Additionally, cyclophosphamide (CYC) $750 \mathrm{mg} / \mathrm{m}^{2}$ of body surface i.v. with urocystitis prophylaxis with mesna was started. CYC was repeated every 4 weeks [15] instead of CYCLOPS protocol because of inconvenience of frequent hospital visits for the patient due to a far distance between his home and the university hospital. Gabapentin was added to ease the neuropathic pain in the forearm and the patient received physiotherapy to improve his fitness and muscle strength. Pneumocystis jirovecii (PJ) prophylaxis with oral cotrimoxazole three times per week was given as well. Prednisolone was tapered by $5 \mathrm{mg}$ every week. During the induction treatment, nausea, chest pain, ocular pain, paraesthesias in the fingers, arthralgias as well as recurrent urticaria on the neck and forearms were reported and ascribed to side effects of
CYC. Furthermore, painful bleeding ulcerations on the tips of unilateral fingers 2 and 3 , associated with cold acra, developed 5-6 months after initiation of the induction treatment. Oscillography detected perfusion defects that improved on sublingual nitroglycerin administration [16]. Antiphospholipid screening was negative. A vasodilatative therapy with iloprost, initially $10 \mu \mathrm{g}$, followed by $20 \mu \mathrm{g}$ was introduced: the first cycle consisting of 7 infusions, the second and third cycle 4 weeks apart each consisting of 5 infusions, followed by further separate infusions every 4 weeks [17].

Controlled MRT, performed 5 months after the first infusion of CYC, showed increasing thickness of the right-side dura mater $(6 \mathrm{~mm})$ and increasing infiltration of the right orbit associated with slight exophthalmus. Infiltration of the parapharyngeal tissue, pterygoid muscle, tissue around the right internal carotid and bilateral obliteration of the mastoid remained unchanged (Fig. 1.).

This condition was classified as progressive disease, and taking into account the intolerance reactions, as mentioned above, the induction therapy was changed after 5 cycles of CYC to rituximab i.v., administered at a dose of $1000 \mathrm{mg}$ two weeks apart and repeated every 6 months [18]. Additionally, prednisolone was increased to $1 \mathrm{mg} / \mathrm{kg}$ body weight again. Four months after this treatment switch, atypical resection of the stomach was performed; the initially suspected GIST lesion was not confirmed, the histologic analysis showed leiomyoma.

Four months after the first cycle of rituximab, MRT controlled examination showed significant reduction of the pachymeningitis and other findings described above; the bilateral obliteration of the mastoid remained stable. After 22 infusions with iloprost, complete remission of digital ulcerations achieved and iloprost was stopped. During rituximab administration, the patient reported arthralgias in large joints, cephalea, fatigue, sleeplessness as well as paraesthesias in the right foot. As the arthralgias did not improve, we assumed a joint manifestation of the underlying disease and adjusted the dose of prednisolone temporarily and added $20 \mathrm{mg}$ of oral methotrexate (MTX) per week, 16 months after initiation of the induction treatment with CYC [19]. MTX was well tolerated, neurologic symptoms on the right foot improved.

After the 3rd cycle of rituximab, further regression of pachymeningitis, tissue infiltrations as well as reduction of exophthalmus were confirmed by MRT examination (Fig. 2 and 3).

The mastoid obliteration remained unchanged. Regression and, finally, remission of meningitis were observed on the MRT scans after the 5th cycle of rituximab. Magnetic resonance angiography did not confirm morphologic changes in the vessels typically seen in cerebral vasculitis [20].

Up to now, our patient has received 7 cycles of rituximab (each comprising two infusions with $1000 \mathrm{mg}$ ). Cotrimoxazole has been discontinued. The patient suffers from intermittent arthralgias. Repeated laboratory examinations did not confirm any pathologic changes with respect to the underlying disease: no changes in blood count, no ANCAs, no inflammation markers, no proteinuria. At the next check-up we are planning to perform cerebral MRT to confirm the sustained remission in order to 


\section{КЛИНИЧЕСКИЕ НАБЛЮДЕНИЯ / CLINICAL OBSERVATIONS}
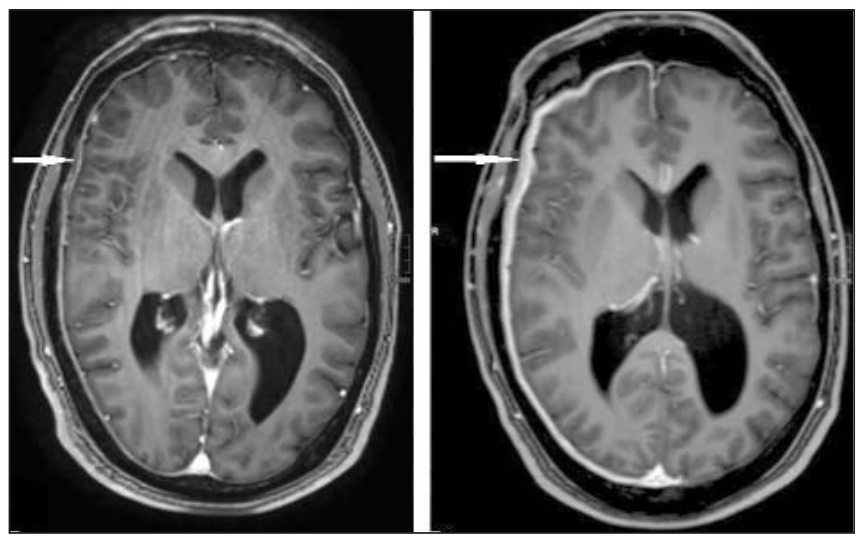

Fig. 2. Cerebral MRT. Regression of pachymeningitis (arrows) after 3 cycles of rituximab (left picture) compared to the situation of progressive disease after 5 cycles of CYC (right picture). Time span between the pictures: 16 months

introduce a maintenance treatment. This will be performed either with MTX or with rituximab for the next 18-24 months, as published and recommended previously [10, 11]. Currently, the patient takes the following medications: methotrexate $20 \mathrm{mg}$ per week, folic acid $5 \mathrm{mg}$ on the 3rd and 4th day after MTX, prednisolone $5 \mathrm{mg}$ per day, vitamin $\mathrm{D}_{3} 30$ drops per week, gabapentin $600 \mathrm{mg}$ three times per day and acetylsalicylic acid $100 \mathrm{mg}$ per day.

\section{Discussion}

We demonstrate a successful treatment of a patient with cANCA-associated vasculitis, who initially presented with otorhinolaryngologic complications and sterile pachymeningitis. Due to missing response to CYC we switched the induction therapy to rituximab that confirmed its positive effects on the above mentioned manifestations, which is in concordance with published observations. This case confirms the experience and recommendations of many physicians that rituximab may be the drug of choice in induction setting of cANCA-associated vasculitis as it shows very good efficacy and better safety than CYC even in atypical manifestations involving the central nervous system.

\section{Conclusions}

We have demonstrated a complete remission on RTX in a patient with cANCA-associated vasculitis and sterile pachymeningitis refractory to 5 cycles of CYC in the induction treatment. RTX has been well tolerated.
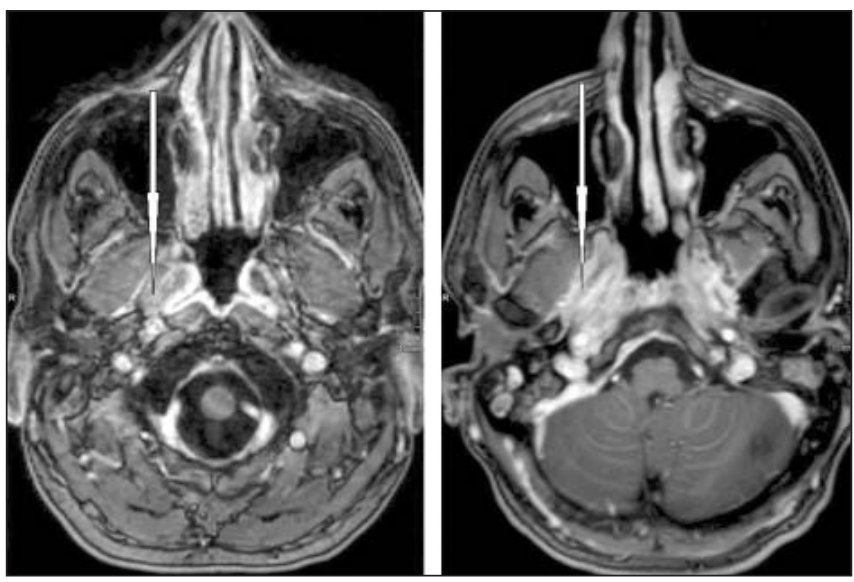

Fig. 3. Cerebral MRT. Regression of the right pterygoid muscle thickness (arrows) after 3 cycles of rituximab (left picture) compared to the situation of progressive disease after 5 cycles of CYC

(right picture). Time span between the pictures: 16 months

\section{Acknowledgments}

Funding

No funding or sponsorship was received for this study or publication of this article.

\section{Authorship}

All named authors meet the International Committee of Medical Journal Editors (ICMJE) criteria for authorship for this article, take responsibility for the integrity of the work as a whole, and have given their approval for this version to be published.

Medical writing, editorial, and other assistance

The authors thank Dr. Marcus Lauschmann, specialist in radiology, for providing images of MRT of our patient.

\section{Disclosures}

Oskar Psenak and Richard Greil declare that they have no conflict of interest.

\section{Ethics statement}

We confirm that the patient provided a written informed consent on publishing his case and images.

Our study did not require an ethical board approval because our patient has been treated with drugs usually used and approved for the treatment of granulomatosis with polyangiitis. No experimental treatment has been used.

\section{R E F}

1. Greco A, Marinelli C, Fusconi M, et al. Clinic manifestations in granulomatosis with polyangiitis. Int J Immunopathol Pharmacol. 2016 Jun;29(2):151-59. doi:10.1177/ 0394632015617063.

2. Tervaert JWC. Trimethoprim-sulfamethoxazole and antineutrophil cytoplasmic antibodies-associated vasculitis. Curr Opin Rheumatol. 2018 Jul;30(4):388-94. doi:10.1097/BOR.0000000000000508. 3. Villa-Forte A, Clark TM, Gomes M, et al. Substitution of methotrexate for cyclophos- phamide in Wegener granulomatosis: a 12-year single-practice experience. Medicine (Baltimore). 2007 Sep;86(5):269-77. doi:10.1097/MD.0b013e3181568ec0. 4. Harper L, Morgan MD, Walsh M, et al. Pulse versus daily oral cyclophosphamide for induction of remission in ANCA-associated vasculitis: long-term follow-up. Ann Rheum Dis. 2012 Jun;71(6):955-60. doi:10.1136/ annrheumdis-2011-200477.

5. Stone JH, Merkel PA, Spiera R, et al. Rituximab versus cyclophosphamide for
ANCA-associated vasculitis. $N$ Engl J Med. 2010 Jul 15;363(3):221-32. doi:10.1056/ NEJMoa0909905.

6. Aydin Z, Gursu M, Karadag S, et al. Role of plasmapheresis performed in hemodialysis units for the treatment of anti-neutrophilic cytoplasmic antibody-associated systemic vasculitides. Ther Apher Dial. 2011 Oct; 15(5):493-98. doi:10.1111/j.1744-9987. 2011.00960.x.

7. Oiwa H, Yamasaki S, Endo K, et al.

Experience with seven Japanese patients with 
КЛИНИЧЕСКИЕ НАБЛЮДЕНИЯ I CLINICAL OBSERVATIONS

antineutrophil cytoplasmic antibody-associated vasculitis treated with remissioninduction therapy with intravenous cyclophosphamide according to the CYCLOPS protocol. Intern Med. 2014; 53(20):2291-96. doi:10.2169/internalmedicine.53.2553

8. Kronbichler A, Jayne DRW, Mayer G. Frequency, risk factors and prophylaxis of infection in ANCA-associated vasculitis. Eur J Clin Invest. 2015 Mar;45(3):346-68. doi:10.1111/eci.12410.

9. Just SA, Knudsen JB, Nielsen MK, Junker P. Wegener's granulomatosis presenting with pachymeningitis: clinical and imaging remission by rituximab. ISRN Rheumatol. 2011;2011: 608942. doi:10.5402/2011/ 608942.

10. Puechal X, Pagnoux C, Perrodeau E, et al. Long-Term Outcomes Among Participants in the WEGENT Trial of Remission-Maintenance Therapy for Granulomatosis With Polyangiitis (Wegener's) or Microscopic Polyangiitis. Arthritis Rheumatol. 2016 Mar; 68(3):690-701. doi:10.1002/art.39450.

11. Guillevin L, Pagnoux C, Karras A, et al.
Rituximab versus azathioprine for maintenance in ANCA-associated vasculitis. $N$ Engl J Med. 2014 Nov 6;371(19):1771-80. doi:10.1056/NEJMoa1404231. 12. de Joode AAE, Sanders JSF, Puechal X, et al. Long term azathioprine maintenance therapy in ANCA-associated vasculitis: combined results of long-term follow-up data. Rheumatology (Oxford). 2017 Nov 1;56(11): 1894-1901. doi:10.1093/rheumatology/ kex281.

13. Qaisar H, Shenouda M, Shariff M, et al. Granulomatosis with Polyangiitis Manifesting as Refractory Otitis Media and Mastoiditis. Arch Iran Med. 2019 Jul 1;22(7):410-13. 14. Koldingsnes W, Nossent JC. Baseline features and initial treatment as predictors of remission and relapse in Wegener's granulomatosis. J Rheumatol. 2003 Jan;30(1):80-8. 15. de Groot K, Adu D, Savage CO; EUVAS (European vasculitis study group). The value of pulse cyclophosphamide in ANCA-associated vasculitis: meta-analysis and critical review. Nephrol Dial Transplant. 2001 Oct; 16(10):2018-27. doi:10.1093/ndt/16.10.2018 . 16. Partsch H. Supplementary instrumental examinations in the most frequently occurring peripheral vascular diseases in general practice. Hautarzt. 1985 Apr;36(4):203-11. 17. Foti R, Visalli E, Amato G, et al. Longterm clinical stabilization of scleroderma patients treated with a chronic and intensive IV iloprost regimen. Rheumatol Int. 2017 Feb; 37(2):245-49. doi:10.1007/s00296016-3582-4.

18. Yusof MY, Vital EM, Das S, et al. Repeat cycles of rituximab on clinical relapse in ANCA-associated vasculitis: identifying $\mathrm{B}$ cell biomarkers for relapse to guide retreatment decisions. Ann Rheum Dis. 2015 Sep; 74(9):1734-38. doi:10.1136/annrheumdis2014-206496.

19. Bramsiepe I, Danz B, Heine R, et al. Primary cutaneous manifestation of Wegener's granulomatosis. Dtsch Med Wochenschr. 2008 Jul;133(27):1429-32. doi:10.1055/s-2008-1081089.

20. Schmidt WA, Both M, Reinhold-Keller E. Imaging procedures in rheumatology: imaging in vasculitis. $Z$ Rheumatol. 2006 Nov;65(7):652-56, 658-61. doi:10.1007/ s00393-006-0107-7.

Received/Reviewed/Accepted

26.05.2021/11.07.2021/17.07.2021

\section{Conflict of Interest Statement}

The investigation has not been sponsored. There are no conflicts of interest. The authors are solely responsible for submitting the final version of the manuscript for publication. All the authors have participated in developing the concept of the article and in writing the manuscript. The final version of the manuscript has been approved by all the authors.

Psenak O. https://orcid.org/0000-0002-7124-9220

Greil R. https://orcid.org/0000-0002-4462-3694 